\title{
Biomimetic Dentistry: Basic Principles and Protocols
}

\author{
Dimitrios Dionysopoulos $^{1^{*}}$, Olga Gerasimidou ${ }^{2}$ \\ ${ }^{I}$ Assistant Professor, Department of Operative Dentistry, Faculty of Dentistry, School of Health Sciences, \\ Aristotle University of Thessaloniki, Thessaloniki 54124, Greece \\ ${ }^{2}$ PhD, Department of Operative Dentistry, Faculty of Dentistry, School of Health Sciences, Aristotle University \\ of Thessaloniki, Thessaloniki 54124, Greece
}

*Corresponding Author: Dimitrios Dionysopoulos, Assistant Professor, Department of Operative Dentistry, Faculty of Dentistry, School of Health Sciences, Aristotle University of Thessaloniki, Thessaloniki 54124, Greece.

\begin{abstract}
Biomimetic dentistry is defined as the science, principles, and techniques of advanced adhesive dentistry respecting the philosophy that to restore sufficiently teeth is necessary to "mimicking life" and understanding the natural tooth in its entirety. The principles of biomimetic dentistry impose introduction of advanced composite restorative materials in clinical practice, which should more respect the nature and integrity of the tooth tissues. There are biomimetic restorative protocols that dental clinicians should follow and are divided into two main groups: a) stress-reducing and b) bond-maximizing protocols. Conservative dentistry and minimal invasive therapy belong to the most recent trends in management of dental caries. Recently, bioactive agents have been introduced in various forms for caries prevention and remineralization of the teeth. In conclusion, there is an increased demand the basic principles of biomimetic dentistry to be acknowledged and followed by the dental clinicians in order to be as minimal invasive as possible intending to preserve the remaining healthy tooth tissues.
\end{abstract}

\section{EDITORIAL}

Biomimetic dentistry is defined as the science, principles, and techniques of advanced adhesive dentistry respecting the philosophy that to restore sufficiently teeth is necessary to "mimicking life" and understanding the natural tooth in its entirety [1]. In particular, biomimetic dentistry promotes the merits of conservative dentistry which intends to the longevity of the natural dentition. The etymological roots of the term "biomimetic" derive from Greek language, since "bios" means "life" and "mimetikos" means "imitative". Biomimetic dental approach enhance the remaining tooth structures avoiding when possible destructive restorative treatments, such as crowns, endodontic therapies, and potential tooth extractions. Behind the concept of biomimetic dentistry lays the education of the dental clinicians in modern caries detection and removal, as well as in conservative restorations of tooth structure that reflects on the higher resistance to wear. Some of the concepts that promote biomimetic dentistry include the conservation of dental pulp, repair or elimination of tooth defects, removal of tooth pathology, saving and strengthening the intact tooth structure, and delaying the re-treatment cycle [2]. During the last decades, the restorative approach has steadily evolved, progressing from mechanical retention to advanced adhesion. Composite resin materials and adhesive dentistry have become valuable tools on this context. The principles of biomimetic dentistry impose introduction of advanced composite restorative materials in clinical practice, which should more respect the nature and integrity of the tooth tissues.

There are biomimetic restorative protocols that dental clinicians should follow and are divided into two main groups: a) stress-reducing and b) bond-maximizing protocols [2]. Stress reducing protocols include: 1) the use of indirect or semidirect restorations for the occlusal and interproximal enamel replacements, 2) the reduction of the increment thickness of the composites $(<2 \mathrm{~mm})$ in dentin surfaces, 3$)$ the incorporation of reinforcing fibers in composite restorations, 4) the selection of slow start or pulse-activated polymerization techniques, 5) the utilization of dentin replacing composites 
with low shrinkage $(<3 \%)$ and range of modulus of elasticity between 12-20 GPa, 6) the use of dual cure composite materials when restoring pulp chambers in non-vital teeth, 7) the removal of dentin cracks within $2 \mathrm{~mm}$ of the dentino-enamel junction, 8) the limitation of onlay cusps to thinner than $2 \mathrm{~mm}$ and 9) the verticalization of occlusal forces by restoring anterior guidance with bonded composite to the lingual surface of maxillary cuspids and the facial surfaces of mandibular cuspids.

Bond-maximizing protocols include [2]: 1) ensurement of a caries-free zone 2-3 mm circumferentially around the cavity without exposing the pulp, 2) repair of composite restorations by applying surface modification techniques on the composite surfaces (airabrasion, silane, etc.), 3) enamel beveling before restoration, 4) deactivation of matrix metalloproteinases (2\% chlorhexidine, etc.), 5) utilization of gold standard dentin bonding systems that can achieve a microtensile bond strength of 25-35 MPa on enamel and 40-60 MPa on flat dentin surfaces, 6) use of immediate dentin sealing (may increase the microtensile bond strength up to $400 \%$ ), 7) resin coating of the immediate dentin sealing using a flowable resin or a lower viscosity restorative composite with a modulus of elasticity of around $12 \mathrm{GPa}$ and 8) elevation of sub-gingival box margins to a supra-gingival position to obtain a biomimetic microtensile bond strength $>30 \mathrm{MPa}$.

Conservative dentistry and minimal invasive therapy belong to the most recent trends in management of dental caries [3]. It has been recognized that new approaches to intervene earlier in the caries process using new technologies should be the most appropriate therapy especially in "high caries risk" children and adults [3, 4]. Proteins of the developing enamel extracellular matrix control initial mineral deposition and subsequent crystal growth, ultimately determining the physicomechanical properties of the mature mineralized tissue [5]. The inorganic content of the hard tooth tissues is made of hydroxylapatite with foreign ions incorporated in the structure (carbonate, fluoride, and magnesium are the most common). In a biomimetic mineralization strategy based on normal enamel histogenesis, a three-dimensional scaffold is formed to attract and arrange calcium $(\mathrm{Ca})$ and/or phosphate $(\mathrm{P})$ ions to induce de novo precipitation of hydroxylapatite [6].
Recently, bioactive agents such as bioactive glass (BAG) 45S5 [7], casein phosphopeptide amorphous calcium phosphate (CPP-ACP) [8] and nano-hydroxyapatite (n-Hap) [9] have been introduced in various forms for caries prevention and remineralization of the teeth. BAG 45S5 is an inorganic amorphous, calcium, sodium phospho-silicate material which contains fivefold ratio of $\mathrm{Ca} / \mathrm{P}$. It interacts with aqueous solutions such as saliva to form a hydroxycarbonate apatite layer, attached chemically to the treated surfaces [10]. BAG application on tooth tissues can release high concentrations of $\mathrm{Ca}$ and $\mathrm{P}$ ions which may be capable of penetrating and remineralizing the sub-surface demineralized enamel via the porous enamel surfaces that was subjected to an acidic attack [11], reducing the erosive activity [12]. CPP-ACP increases the number of potential calcium-binding sites on enamel surface leading to reduction of the constant diffusion of calcium. Additionally, ACP buffers the free calcium and phosphate ion activities on enamel surface, assisting to maintain a state of supersaturation that reduces demineralization and enhances remineralization [13]. Moreover, the application of CPP-ACP on enamel surface facilitates the formation of a crystal layer, which fills the interprism voids and partially covers the apatite prisms protecting them from the acidic activity [14]. Synthetic n-HAp is composed of apatite crystals in size of $50-1000 \mathrm{~nm}$ and constitutes a perfect substitute for enamel microstructure, especially in acidic environment. It is a source of $\mathrm{Ca}$ and $\mathrm{P}$ ions and forms strong bond with enamel surface [15].

In conclusion, nowadays there is an increased demand the basic principles of biomimetic dentistry to be acknowledged and followed by the dental clinicians in order to be as minimal invasive as possible intending to preserve the remaining healthy tooth tissues.

\section{REFERENCES}

[1] Magne P. Esthetic and Biomimetic Restorative Dentistry: Manual for Posterior Esthetic Restorations. Los Angeles, CA: USC School of Dentistry (2006).

[2] Alleman D.S., Matthew A., Alleman D.S. The Protocols of Biomimetic Restorative Dentistry: 2002 to 2017. Increase the longevity of restorations with the biomimetic approach, Inside Dent. 13, (2017).

[3] Cummins D., The development and validation of a new technology, based upon $1.5 \%$ 
arginine, an insoluble calcium compound and fluoride, for everyday use in the prevention and treatment of dental caries. J. Dent. 41, 1-11 (2013).

[4] Frenken J.E., Peters M.C., Manton D.J., Leal S.C., Gordan V.V., Eden E., Minimal intervention dentistry for managing dental caries - a review: report of a FDI task force. Int. Dent. J. 62, 223-243 (2012).

[5] Simmer J.P., Fincham A.G. Molecular Mechanisms of Dental Enamel Formation. Crit. Rev. Oral Biol. Med. 6, 84-108 (1995).

[6] Palmer L.C., Newcomb C.J., Kaltz S.R., Spoerke E.D., Stupp S.I. Biomimetic systems for hydroxyapatite mineralization inspired by bone and enamel. Chem. Rev. 108, 4754-4783 (2008).

[7] Dionysopoulos D., Tolidis K., Sfeikos T. Effect of air-abrasion pre-treatment with bioactiveglass $45 \mathrm{~S} 5$ on enamel surface loss aftererosion/abrasion challenge. Dent. Mater. 35, 193-203 (2019).

[8] Wang C.P., Huang S.B., Liu Y., Li J.Y., Yu H.Y. The CPP-ACP relieved enamel erosion from a carbonated soft beverage: An in vitro AFM and XRD study. Arch. Oral Biol. 59, 277-282 (2014).

[9] Dionysopoulos D., Tolidis K., Sfeikos T. Effect of CPP-ACPF and nanohydroxyapatite preventive treatments on the susceptibility of enamel to erosive challenge. Oral Health Prev. Dent. 17, 357-364 (2019).

[10] Thompson I.D., Hench L.L. Mechanical properties of bioactive glasses, glass-ceramics and composites. Proc. Inst. Mech. Eng. H. 212, 127-136 (1998).

[11] Bakry A.S., Marghalani H.Y., Amin O.A., Tagami J. The effect of a bioglass paste on enamel exposed to erosive challenge. J. Dent. 42, 1458-1463 (2014).

[12] Dionysopoulos D., Tolidis K., Tsitrou E., Kouros P., Naka O. Quantitative and qualitative evaluation of enamel erosion following air-abrasion with bioactive glass 45S5. Oral Health Prev. Dent. 18, 529-536 (2020).

[13] Ranjitkar S., Kaidonis J.A., Richards L.C., Townsend G.C. The effect of CPP-ACP on enamel wear under severe erosive conditions. Arch. Oral Biol. 54, 527-532 (2009).

[14] Poggio C., Lombardini M., Dagna A., Chiesa M., Bianchi S. Protective effect on enamel demineralization of a CPP-ACP paste: an AFM in vitro study. J. Dent. 37, 949-954 (2009).

[15] Huang S., Gao S., Yu H. Remineralization potential of nano-hydroxyapatite on initial enamel lesions: an in vitro study. Caries Res. 45, 460-468 (2011).

Citation: Dimitrios Dionysopoulos, Olga Gerasimidou. Biomimetic Dentistry: Basic Principles and Protocols. ARC Journal of Dental Science. 2020; 5(3):1-3. DOI: https://doi.org/10.20431/2456-0030.0503001.

Copyright: (C) 2020 Authors. This is an open-access article distributed under the terms of the Creative Commons Attribution License, which permits unrestricted use, distribution, and reproduction in any medium, provided the original author and source are credited. 